\title{
The Model of Cost Optimization in Sales Process
}

\author{
Xin-Yi CHEN ${ }^{1, a,{ }^{*}}$ \\ ${ }^{1}$ China Minorities Information Technology Institute, Northwest University for Nationalities, Lanzhou, \\ Gansu, China \\ acxyxbmd@sina.com \\ ${ }^{*}$ Corresponding author
}

Keywords: stochastic optimization, sales, sales period, penalty function.

\begin{abstract}
This paper puts forward a method for optimizing sales cost of a special kind of sales with randomicity. This method transforms stochastic optimization into a determinable one. It has two steps: first, by the means of mean sales amount, the problem is transformed into a discrete variate optimization one without randomicity, second, by the means of penalty function method, the discrete variate optimization problem is transformed into a continuous variate optimization problem. This method is expected to be effective for sales cost optimization of wholesale.
\end{abstract}

\section{Introduction}

Randomicity exists in many retail processes, and it takes trouble to optimize retail cost. In the last few years, there are many papers to deal with the influence of randomicity on sales process[1], by building and using probabilistic models, showing us some stochastic optimization methods with constraints.

As for sales question, stochastic optimization is also a research hotspot. This paper deals with a kind of question of these, it has properties

1) In a short time interval, the sales amount of some kind of retail has strong randomicity.

2) In a relative long time interval with some start time, the sales amount of the retail fluctuates around a constant with very small swing.

3) For two time intervals with same length but different start time, the two constants (the referred in 2), around which two sales amount fluctuate respectively, are different.

\section{Description of the Problem}

In the sales process of some kind of merchandise, for some reason, it is can not be ensured that every merchandise is sold. So the customer can select suitable ones from a large quantity of merchandises. In this kind of sales process, the quantity of suitable one in few merchandises is not a constant, but with the quantity of merchandise increasing, the merchandise of suitable one to total number (both suitable ones and unsuitable ones included) changes regularly. The merchandise sale percent of the first 50 merchandises after shopping mall selling is $20 \%$, with customer recognition increasing, the merchandise sale percent of the second 50 merchandises after shopping mall selling is different from the first one and it is $30 \%$, the third one, the forth one, and so on, are different with each other, but every merchandise sale percent of 50 merchandises fluctuates around a certain constant. If the time of selling one merchandise (it may be a suitable one or may be not) or the mean time of sold a large quantity of merchandises is a constant, the product sold percent of the merchandise in a relatively long period changes regularly with the time. If the sale speed is 25 merchandise pre hour, the quantity of suitable merchandise of the first two hours after shopping mall selling is 10 , the one of the second hour is 15 , the third one, the forth one, and so on are different with each other, but the quantity of suitable merchandise of respective period is steady. This paper brings forwards a method to deal with the question with the randomicity mentioned above. The idea of this method is using the stability of mean sales amount of long period to decrease the difficulty of sale cost optimization which is brought by randomicity of sales amount of short period.

There are several different sales assistants who can sell the same merchandise in a shopping mall, every sales assistant's amount is different from others. For every sales assistant, his sales amount of short period 
has stronger randomicity, but its mean sales amount of long period fluctuate around a constant with small swing, and the constant have something to do with the start time. We have following constraints:

1) The sales assistant must halting after a period of selling to rest, that is the selling time can not be unlimited.

2) Because of the influence of some factors, the selling quantity of every sales assistant has an upper limit.

3) Different sales assistant has different maximum selling time and different maximum halt time.

4) Different sales assistant has different upper limit of selling quantity.

The objective of the sales problem is that, by reasonably scheduling the selling times and the halting times of all sales assistant, minimize the sales cost.

\section{Main Results}

\section{A. Model with Discrete Variate}

Objective function:

1) The objective function is given by

$$
\min z=\sum_{j=1}^{T} \sum_{i=1}^{N} F_{i, j}\left(P_{i, j}, x_{i, j}, t_{i, j}^{s e}, t_{i, j}^{h a}\right)
$$

for $i=1,2, \cdots, N$, and $j=1,2, \cdots, T$, where $z$ is the sale cost of a plan period.

$i=1,2, \cdots, N$, and $N$ is the quantity of sales assistant in the shopping mall, $j=1,2, \cdots, T$ and $T$ is the quantity of sale period in one plan period, $P_{i, j}$ is the sales amount of the $i$ th sales assistant in the $j$ th sale period, $x_{i, j}$ is the state (selling or halting) of the $i$ th sales assistant in the $j$ th sale period, $t_{i, j}^{s e}$ is the continuous selling time of the $i$ th sales assistant to the $j$ th sales period, $t_{i, j}^{h a}$ is the continuous halting time of the $i$ th sales assistant to the $j$ th sales period, $F_{i, j}$ is the operation cost of the $i$ th sales assistant to the $j$ th sales period, it is the function of $P_{i, j}, x_{i, j}, t_{i, j}^{s e}$ and $t_{i, j}^{h a}$.

The objective is optimizing the sales cost $z$.

2) Constraint Condition:

Constraint conditions ensure the continuous and steady sale. This problem includes two kinds of constraint conditions: random variate constraints and determinable variate constraints, and the determinable variate constraints also include two kinds of constraints: steady constraints and dynamic constraints. Steady constraints are the constraints that are only related to a single sales period, but the dynamic constraints are the constraints that are related to more than one sales periods.

a) Random variate constraint

The random variate constraint of this problem is the constraint on variate $P_{i, j}$, it is also the constraint on the upper limit and lower limit of the sales amount of the sales assistants. For the sales cost optimization with determinable sales amount, the value of, in a sales period with each given length can be determined, but for this problem, a relative steady value of can be determined only when the sales period length is large enough. Let $P_{i, j}^{\max }$ be the upper limit of $P_{i, j}$ and $P_{i, j}^{0}$ be the relative steady value of $P_{i, j}$. In general, the value selected of $P_{i, j}^{\max }$ should less than $P_{i, j}^{0}$ appropriately, thus the production target can be accomplished with high probability. So we constraint the $P_{i, j}$ as follow:

$0 \leq P_{i, j} \leq P_{i, j}^{\max }$

for $i=1,2, \cdots, N$ and $j=1,2, \cdots, T$.

Thus, this strong randomicity problem is transformed into a weak randomicity one. If $P_{i, j}^{\max }$ selected is small enough, this problem can be transformed into a determinable one. 
Determinable Variate Constraints

b) Steady Constraints

1) Constraints on Supply and Demand

$$
\sum_{i=1}^{N} x_{i, j} P_{i, j}=P_{\sigma, j}
$$

for $i=1,2, \cdots, N$ and $j=1,2, \cdots, T$, where $P_{\sigma, j}$ is the whole sales amount of the shopping mall in the $j$ th sale period.

2) Constraints on Sales Ability for Emergency

$$
\sum_{i=1}^{N} x_{i, j} P_{i, j}^{\max }-P_{\sigma, j} \geq S
$$

for $i=1,2, \cdots, N$ and $j=1,2, \cdots, T$, where $S$ is the emergency sales ability (mentioned above) that shopping mall remains.

Dynamic Constrains

Dynamic constraints of the problem are the constraint on selling times and halting times of sales assistant, they are

$$
\left\{\begin{array}{l}
t_{i, j}^{s e} \leq T_{i, \max }^{s e} \\
t_{i, j}^{h a} \leq T_{i, \max }^{h a}
\end{array}\right.
$$

for $i=1,2, \cdots, N$ and $j=1,2, \cdots, T$, where $P_{i, \max }^{s e}$ is the maximum selling time of the $i$ th sales assistant and,$P_{i, \max }^{h a}$ is the maximum halting time of the $i$ th sales assistant. The constraints on selling times and halting times in the (5) are general expression. In order to accurate computation, it needs to be described in detail. Selling times and halting times accelerate as time goes on, so, selling time and halting times can be expressed as

$$
\left\{\begin{array}{l}
t_{i, j}^{s e}=\left(t_{i, j-1}^{s e}+T_{j}\right) x_{i, j} \\
t_{i, j}^{h a}=\left(t_{i, j}^{h a}+T_{j}\right)\left(1-x_{i, j}\right)
\end{array}\right.
$$

and (5) can be written as

$$
\left\{\begin{array}{l}
\left(t_{i, j-1}^{s e}+T_{j}\right) x_{i, j}-T_{i, \text { max }}^{s e} \leq 0 \\
\left(t_{i, j}^{h a}+T_{j}\right)\left(1-x_{i, j}\right)-T_{i, \text { max }}^{h a} \leq 0
\end{array}\right.
$$

for $i=1,2, \cdots, N$ and $j=1,2, \cdots, T$, where $T_{j}$ is the length of $j$ th sale period. So $t_{i, j}^{s e}$ and $t_{i, j}^{h a}$ are functions of $x_{i, j}$.

Thus we obtain a mathematical model of non-linear maxed 0-1 bivariate integer optimization, and it can be described as following

Objective Function

$$
\min z=\sum_{j=1}^{T} \sum_{i=1}^{N} F_{i, j}\left(P_{i, j}, x_{i, j}, t_{i, j}^{s e}, t_{i, j}^{h a}\right)
$$

for $i=1,2, \cdots, N$ and $j=1,2, \cdots, T$.

Constraint Conditions 


$$
\begin{aligned}
& \{0, \text { if sales assistant is halting } \\
& 1, \quad \text { if sales assistant is selling } \\
& 0 \leq P_{i, j} \leq P_{i, j}^{\max } \\
& \sum_{i=1}^{N} x_{i, j} P_{i, j}=P_{\sigma, j} \\
& \sum_{i=1}^{N} x_{i, j} P_{i, j}^{\max }-P_{\sigma, j} \geq S \\
& \left\{\begin{array}{l}
t_{i, j}^{s e}=\left(t_{i, j-1}^{s e}+T_{j}\right) x_{i, j} \\
t_{i, j}^{h a}=\left(t_{i, j}^{h a}+T_{j}\right)\left(1-x_{i, j}\right)
\end{array}\right. \\
& \left\{\begin{array}{l}
\left(t_{i, j-1}^{s e}+T_{j}\right) x_{i, j}-T_{i, \text { max }}^{s e} \leq 0 \\
\left(t_{i, j}^{h a}+T_{j}\right)\left(1-x_{i, j}\right)-T_{i, \max }^{h a} \leq 0
\end{array}\right.
\end{aligned}
$$

for $i=1,2, \cdots, N$ and $j=1,2, \cdots, T$.

B. Model with Continuous Variate

Penalty function method is an important method for solving non-linear constraint optimization. By this method, some discrete viriate constraints can be transformed into continuous ones [2, 3]. We next transform the model with discrete variate into a model of continuous variate by adopting integer optimization penalty function method. Relax the scale of $x_{i, j}$

$$
0 \leq x_{i, j} \leq 1
$$

for $i=1,2, \cdots, N$ and $j=1,2, \cdots, T$. Then add penalty item into objective function:

$$
M x_{i, j}\left(1-x_{i, j}\right)
$$

for $i=1,2, \cdots, N$ and $j=1,2, \cdots, T$. where $M$ is a very large positive integer. The last objective function is

$$
\min z=\sum_{j=1}^{T} \sum_{i=1}^{N}\left[F_{i, j}+M x_{i, j}\left(1-x_{i, j}\right)\right]
$$

for $i=1,2, \cdots, N$ and $j=1,2, \cdots, T$.

The last model with continuous variate is

Objective Function

$$
\min z=\sum_{j=1}^{T} \sum_{i=1}^{N}\left[F_{i, j}+M x_{i, j}\left(1-x_{i, j}\right)\right]
$$

for $i=1,2, \cdots, N$ and $j=1,2, \cdots, T$.

Constraint Conditions

$$
\begin{aligned}
& 0 \leq x_{i, j} \leq 1 \\
& 0 \leq P_{i, j} \leq P_{i, j}^{\max } \\
& \sum_{i=1}^{N} x_{i, j} P_{i, j}=P_{\sigma, j} \\
& \sum_{i=1}^{N} x_{i, j} P_{i, j}^{\max }-P_{\sigma, j} \geq S
\end{aligned}
$$




$$
\begin{aligned}
& \left\{\begin{array}{l}
t_{i, j}^{s e}=\left(t_{i, j-1}^{s e}+T_{j}\right) x_{i, j} \\
t_{i, j}^{h a}=\left(t_{i, j}^{h a}+T_{j}\right)\left(1-x_{i, j}\right)
\end{array}\right. \\
& \left\{\begin{array}{l}
\left(t_{i, j-1}^{s e}+T_{j}\right) x_{i, j}-T_{i, \text { max }}^{s e} \leq 0 \\
\left(t_{i, j}^{h a}+T_{j}\right)\left(1-x_{i, j}\right)-T_{i, \text { max }}^{h a} \leq 0
\end{array}\right.
\end{aligned}
$$

for $i=1,2, \cdots, N$ and $j=1,2, \cdots, T$.

Now, the problem becomes an non-linear optimization problem of continuous variate. So we can deal with it by methods that deal with non-linear continuous variate optimization.

\section{References}

[1] Pelikan.Martin, Goldberg.David.E, and Lobo.Fernando.G, A survey of optimization by building and using probabilistic models. Mobile Computing. 4(2005) 458-473.

[2] Huang.Qiu-Hong, Cao.De-Xin, and Deng.Ka-Zhong, Penalty function interval method for solving constrained continuous minimax problem, Journal of China University of Mining and Technology. 34(2005) 129-132.

[3] Wang C.Y., Yang X.Q, and Yang X.M, Nonlinear lagrange duality theorems and penalty function methods in continuous optimization. Journal of Global Optimization. 27(2003) 473-484. 